\title{
Perree
}

Juan David Sempere

Souvannavong

\section{Les pieds-noirs à Alicante}

In: Revue européenne de migrations internationales. Vol. 17 №3. pp. 173-198.

Citer ce document / Cite this document :

Sempere Souvannavong Juan David. Les pieds-noirs à Alicante. In: Revue européenne de migrations internationales. Vol. 17 N³. pp. 173-198.

doi : 10.3406/remi.2001.1800

http://www.persee.fr/web/revues/home/prescript/article/remi_0765-0752_2001_num_17_3_1800 


\section{Abstract}

The pieds-noirs in Alicante.

Juan David Sempre Souvannavong.

Alicante is one of the closest european harbours to North Africa. This explains why the relationships with the Maghreb, and especially with Western Algeria, have been so intense and controversial, The period of the independence of Algeria (1962) has been one of the most complex in this relationship. It forced most of the french people native of Europe who were living in the colony, to go into exil. Some of these pieds-noirs, most of them with Spanish origins, decided to settle in Spain, often in Alicante. At this time, Alicante is a small province capital which is starting to open to turism and foreign investment, after more than two decades of autarky. The arrival of several thousands of pieds-noirs strengthens the beginnings of modernisation in the region. Despite an integration with a lot of nuances, during the first years, the pieds-noirs have a decisive influence on the town's economy and society, and to a lesser extend, on demography. They establish in almost all the sectors of the local economy and stand out by reason of the innovative businesses they start as well as by the habits they introduce in some sectors of a society which is still traditional. The massive arrival of turists and european residents (especially retired persons) during the seventies imply a decrease in their influence. However, Alicante still maintain close relations with France.

\section{Résumé}

Les pieds-noirs à Alicante.

Juan David Sempre Souvannavong.

Alicante est un des ports européens les plus proches de l'Afrique du Nord ce qui explique les relations intenses et controversées qu'il a entretenu avec le Maghreb et particulièrement avec l'ouest de l'Algérie. Un des moments les plus complexes de cette relation est la période de l'indépendance de l'Algérie (1962) qui force à l'exil la plupart des Français d'origine européenne qui vivaient dans la colonie. Une petite partie de ces pieds-noirs, la plupart d'origine espagnole, choisissent pour diverses raisons de s'établir en Espagne, souvent dans la province d'Alicante. La ville d'Alicante est à l'époque une petit capitale de province qui s'ouvre, après plus de deux décennies d'autarcie, au tourisme et aux investissements étrangers. L'arrivée de plusieurs milliers de pieds-noirs consolide l'amorce de la modernisation dans la région. Malgré une intégration pleine de nuances, les pied-noirs ont pendant les premières années qui suivent leur arrivée une influence décisive sur l'économie, la société, et en moindre mesure la démographie de la ville. Ils s'établissent presque dans tous les secteurs de l'économie locale, et se distinguent surtout par le nombre de commerces innovateurs qu'ils ouvrent et par les nouvelles habitudes qu'ils introduisent dans certains secteurs d'une société encore traditionnelle. Après l'arrivée en masse de touristes et de résidents européens (surtout des retraités), à partir des années soixante-dix, l'influence des Pied-noirs diminue. En dépit de cela Alicante conserve toujours d'étroites relations avec la France.

\section{Resumen}

Los pieds-noirs en Alicante. Juan David Sempre Souvannavong.

Alicante es uno de los puertos europeos más próximos del Norte de África lo que explica las intensas y controvertidas relaciones que siempre ha mantenido con el Magreb y en particular con el oeste de Argelia. Uno de los momentos más complejos se da cuando la independencia de Argelia (en 1962) fuerza el exilio de la gran mayoría de los franceses de origen europeo que vivían en la colonia. Una pequeña parte de esos pieds-noirs, de antepasados españoles en su mayoría, eligen por diversas razones establecerse en España, a menudo en la provincia de Alicante. La ciudad de Alicante es en la época una pequeña capital de provincia que se abre, tras más de dos décadas de autarquía, al turismo y a las inversiones extranjeras. La llegada de varios miles de pieds-noirs dio un fuerte impulso a la incipiente modernización de la región. A pesar de su integración llena de matices los pieds-noirs tienen, durante los primeros años, una influencia decisiva en la economía, en la sociedad y en menor medida en la demografía de la ciudad. Se establecen en casi todos los sectores de la economía local distinguiéndose sobre todo por el gran número de comercios innovadores que abren por los nuevos hábitos que introducen en ciertos sectores de una sociedad aún tradicional. La llegada en masa de 
turistas y residentes europeos (sobre todo jubilados) a partir de los años setenta diluye la influencia de los pied-noirs. A pesar de ello Alicante sigue manteniendo estrechas relaciones con Francia. 


\section{Les pieds-noirs à Alicante}

\section{Juan David SEMPERE SOUVANNAVONG*}

À la veille de l'indépendance, l'Algérie comptait plus de 1,2 millions de personnes d'origine non africaine ce qui représentait environ $12 \%$ de sa population totale. Cette forte présence d'Européens la différenciait des autres colonies et protectorats français où leur présence était bien moins importante. Espagnols et Français d'origine espagnole constituaient la principale minorité au sein de la société européenne de l'Algérie française, une société qui était le produit des multiples flux migratoires provenant de nombreuses régions de la Méditerranée occidentale depuis le début de la conquête française en 1830 .

L'indépendance de cette colonie, en 1962, a été proclamée après huit années de durs combats, et ces conditions difficiles ont entraîné l'exode vers la métropole d'une grande partie de la population européenne, ceux que l'on appelait les pieds-noirs. Les derniers mois de l'Algérie française et les conditions de l'exil ont profondément marqué la mémoire de ces personnes dont la plupart considérait l'Algérie comme leur pays. La France, qui connaissait depuis une décennie des retours liés à la décolonisation, fut totalement débordée par l'arrivée massive et brutale de près d'un million de rapatriés en 1962. Mais ce mouvement colossal n'a pas touché uniquement la France, d'autres régions ont subi également les effets de ce phénomène : l'Argentine, Israël, le Canada et surtout l'Espagne ont été, d'après Palacio (1968), la destination de nombreux pieds-noirs. Par sa proximité et l'importance de ses relations avec l'Algérie, l'Espagne a été le pays choisi par plusieurs milliers d'entre eux pour refaire leur vie. La plupart sont arrivés dans la province levantine ${ }^{1} \mathrm{~d}$ 'Alicante.

* Departamento de Geografía Humana, Universidad de Alicante, Apdo. Correos, 99, 03080 Alicante, Espana et UMR 6588 Migrinter, Poitiers, e-mail:jd.sempere@ua.es.

1 En Espagne on appelle el Levante la région qui regroupe les provinces méditerranéennes du sud-est du pays (Castellón, Valence, Alicante, Murcie et même Alméria). Elle ne correspond à aucune unité administrative mais elle coïncide avec les zones d'origine de l'émigration espagnole vers l'Algérie au XIX" et au XX" siècle. 
Alicante a été le principal lieu de réinstallation des Français d'Algérie hors de France. Un nombre incertain mais important de réfugiés (de 30 à 40000 selon les reportages de presse maximalistes) y ont refait leur vie participant pleinement à l'évolution de la capitale régionale et de sa province. Ils ont représenté la première vague d'immigration externe dans la région, juste pendant les années où celle-ci commençait à s'ouvrir naïvement à la modernisation et se mettait à l'heure du tourisme. Comme dans de nombreuses régions du Midi français les pieds-noirs ont contribué à Alicante, de façon notoire, à des changements sociaux, urbains et économiques dont les conséquences subsistent encore de nos jours; ceci a souvent provoqué les critiques de ceux qui les percevaient comme les catalyseurs des métamorphoses fortement contestées de l'époque.

Malgré l'importance indéniable de ce phénomène, peu de recherches ont été engagées sur les pieds-noirs à Alicante alors qu'en France, les rapatriements de la décolonisation demeurent un sujet de controverse et font l'objet de nombreuses études et publications.

\section{ÉTÉ 1962 : L'ARRIVÉE DE PLUSIEURS MILliers DE RÉFUGIÉS}

L'arrivée à Alicante des premiers Français fuyant le conflit colonial se note dès 1955 (fig. 1) : au cours de cette année, en Algérie, la guerre se généralise dans l'ensemble du pays. Elle s'intensifie en 1957 entraînant le départ de nombreux piedsnoirs, vivant pour la plupart, en milieu rural. Les exilés républicains de la Guerre Civile espagnole sont souvent les premiers à revenir en Espagne, leur faible attachement au pays et le souvenir d'un conflit encore récent leur a permis de prévoir rapidement le destin incertain de la colonie.

Figure 1 : Année d'arrivée des pieds-noirs recensés à Alicante en 1965

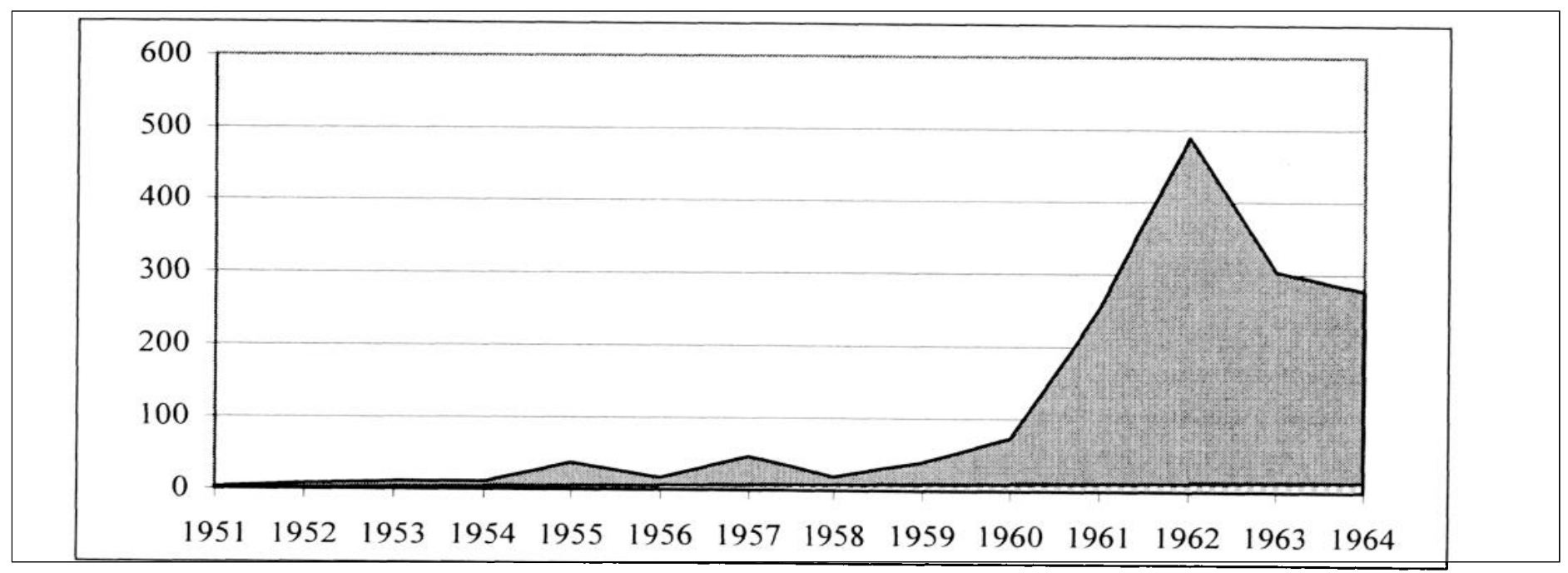

Source : Padrón Municipal de Habitantes de 1965, réalisation personnelle 
Mais ce n'est que pendant la dernière année d'une guerre - pourtant militairement gagnée par l'armée française - que les départs ont véritablement repris (fig. 1). L'année 1961 est celle de l'ouverture des pourparlers entre le gouvernement français et le Front de Libération Nationale (FLN) et de la rupture définitive entre les partisans et les détracteurs de l'Algérie française. C'est à ce moment que des piedsnoirs, prévoyant des moments difficiles, ont quitté l'Algérie ou au moins organisé le départ de leur famille et leurs biens. D'autres, malgré l'indépendance imminente de la colonie, croyaient pouvoir rester dans leur pays natal, ce que prévoyaient les Accords d'Evian ; le FLN a d'ailleurs reconnu l'importance que pouvait avoir leur rôle dans la construction de l'Algérie indépendante (Stora, 1993: 77). Il est vrai que beaucoup de ces anciens colons auraient difficilement pu supporter la République arabisante, socialiste et surtout unitaire que deviendra l'Algérie après l'indépendance. Pourtant, la première cause du départ massif de la population européenne n'a pas été la peur du nouveau régime ou l'idée apocalyptique d'une Algérie indépendante, mais la radicalisation du conflit, la brutalité et la dégradation de la situation au cours des derniers mois de guerre. La plupart des Européens ont compris à ce moment que la réconciliation n'était désormais plus envisageable ce qui a provoqué un rapatriement improvisé et précipité dont l'ampleur a dépassé toutes les prévisions gouvernementales? ${ }^{2}$.

À Alicante, comme en France, les arrivées de réfugiés se multiplient dès le début du printemps 1962. Il semble que le déclenchement de l'immigration soit lié notamment au voyage organisé par la mairie d'Alicante pour participer à la Foireexposition d'Oran de cette année. Les principales personnalités de la ville se rendent en Algérie et beaucoup de pieds-noirs profitent de leur voyage de retour et de la complicité des autorités espagnoles ${ }^{3}$ pour partir en Espagne avec leurs biens. Pendant les mois d'avril et de mai la ville reçoit par l'intermédiaire des deux bateaux français de sa ligne régulière avec Oran, un nombre croissant de personnes ${ }^{4}$. 11 s'agit évidemment d'un nombre d'arrivées moins considérable qu'en France mais la migration est cncore plus spontanée et plus désorganisée. Au cours des semaines qui précèdent l'indépendance, le départ de plus en plus massif des pieds-noirs tourne à l'exode, c'est alors que bon nombre d'entre cux font le choix d'Alicante. À la fin du mois de juin 1962 le gouvernement espagnol envoie à Oran deux bateaux escortés par des bâtiments de guerre pour rapatrier les ressortissants espagnols. Leur retour, aux

2 Le 26 décembre 1961 le gouvernement français promulga une loi sur les rapatriés $\left(n^{\circ} 61.1439\right.$ ) qui prévoyait l'arrivée de 100000 pieds-noirs en 1962 et de 400000 pendant les quatre années suivantes. Au 31 mai 1962 les entrées en France dépassaient déjá les 275000 personnes, pendant le mois de juin débarquèrent 354914 passagers (Jordi : $1992: 27$ ).

3 "Je crois - a signalé Agatángelo Soler (le Maire d'Alican-te de 1959 à 1965) - que nous avons rapporté tout l'argent du pays (...). Le Gouverneur Civil ll'équiralent du Préfét e'n France) et moi-même nous arons dû distraire les autorités douanièrés pour qu' elles né s'apersoivent pas de l'embarquement. Je me somiens qu' une des ialises s'ourrit et tout l'argent tomba par terre, le Gowernéur lui-môme aida à ramasser l'argent". Daprès le quotidien Información du 2 février 1992.

4 D’après le quotidien Información. 6968 réfugiés ont débarqué à Alicante pendant le mois de juin 1962, mais ce chiffre n'est pas sûr puisque le journal ne précise que rarement le nombre exact de personnes arrivées. 
ports de Carthagène et d'Alicante, avec plus de 4000 réfugiés à bord fait enfin la Une du quotidien Información, premier journal local à avoir couvert, très discrètement, l'arrivée des réfugiés au cours des mois précédents. Alicante et Carthagène sont les ports européens les plus proches de l'Algérie occidentale, et il est fréquent que ceux qui ne parviennent pas à s'embarquer sur les bateaux des lignes régulières vers la France atteignent alors les ports du sud-est de l'Espagne, souvent dans des embarcations de fortune et dans les situations les plus désespérées. Les tout derniers défenseurs de l'Algérie française seraient ainsi arrivés à Alicante (Palacio, 1968:41) le $1^{\text {er }}$ juillet 1962.

Alicante apparaît alors comme un lieu d'exil proche et tranquille où se concentrent spontanément tous les pieds-noirs arrivés en Espagne sans qu'il y ait eu d'ordre de réunion générale. Beaucoup ont des parents dans la région, d'autres espèrent un apaisement du conflit pour revenir en Algérie. Mais, après l'indépendance, les luttes intestines pour le pouvoir, le massacre des harkis et la nationalisation par le nouveau gouvernement algérien des biens abandonnés prouvent rapidement l'impossibilité du retour rêvé. L'Algérie deviendra alors le paradis perdu auquel Alicante par son climat et son ambiance ressemble trop. Il est vrai que pendant les premiers mois une partie des réfugiés quittent l'Espagne pour aller travailler ou étudier en métropole, mais d'autre part un grand nombre d'anciens colons arrivent d'Algérie, du Maroc ou de France et s'installent à Alicante où commence à s'organiser une forte communauté pied-noire.

\section{POURQUOI L'ESPAGNE ? POURQUOI ALICANTE ?}

L'histoire des échanges entre la région d'Oran et le Levant espagnol est longue, facilitée par la proximité. Dans le passé les deux régions ont souvent dépendu de la même administration; jusqu'en 1830 Alicante a été une des destinations de choix pour les razzias de pirates nord-africains; depuis se sont succédé des périodes de fortes relations entre les populations de l'Oranie et du Levant espagnol et d'autres pendant lesquelles les difficultés politiques les ont interrompu. La dernière rupture a été celle de la Guerre Civile espagnole (1936-39), et les relations ont repris avec force au début des années cinquante. Certaines des raisons qui expliquent ce lien permettent également de justifier l'établissement des pieds-noirs à Alicante.

\section{Proximités et similitudes}

Les affinités naturelles et humaines qui rapprochent ces deux rives de la Méditerranée méritent d'être présentées comme une raison importante pour laquelle de nombreux pieds-noirs ont décidé de s'installer à Alicante. Ces similitudes découlent de la proximité géographique des deux régions : en effet, les villes d'Oran et d'Alicante sont séparées par moins de 250 kilomètres qui peuvent être parcourus en douze heures en voilier. Malgré de grandes différences culturelles, les deux régions ont une histoire commune et appartiennent indéniablement à un même ensemble méditerranéen ; le paysage de la province d'Alicante, la ville même, ressemblent étrangement à Oran et à sa région. Les villages, certaines coutumes et surtout le climat et la luminosité sont très semblables de part et d'autre de la mer. 
Beaucoup de pieds-noirs évoquent précisément le climat, le soleil et la Méditerranée pour justifier leur choix de cette région de résidence. Ce sont ces similitudes avec leur environnement natal, qu'ils n'ont pas réussi à trouver en France, qui expliquent, dans ce contexte particulier qu'est l'exil, l'attraction qu'Alicante a exercé sur un grand nombre d'anciens habitants européens d'Afrique du Nord.

Figure 2 : La proximité entre l'Ouest algérien et le Levant espagnol

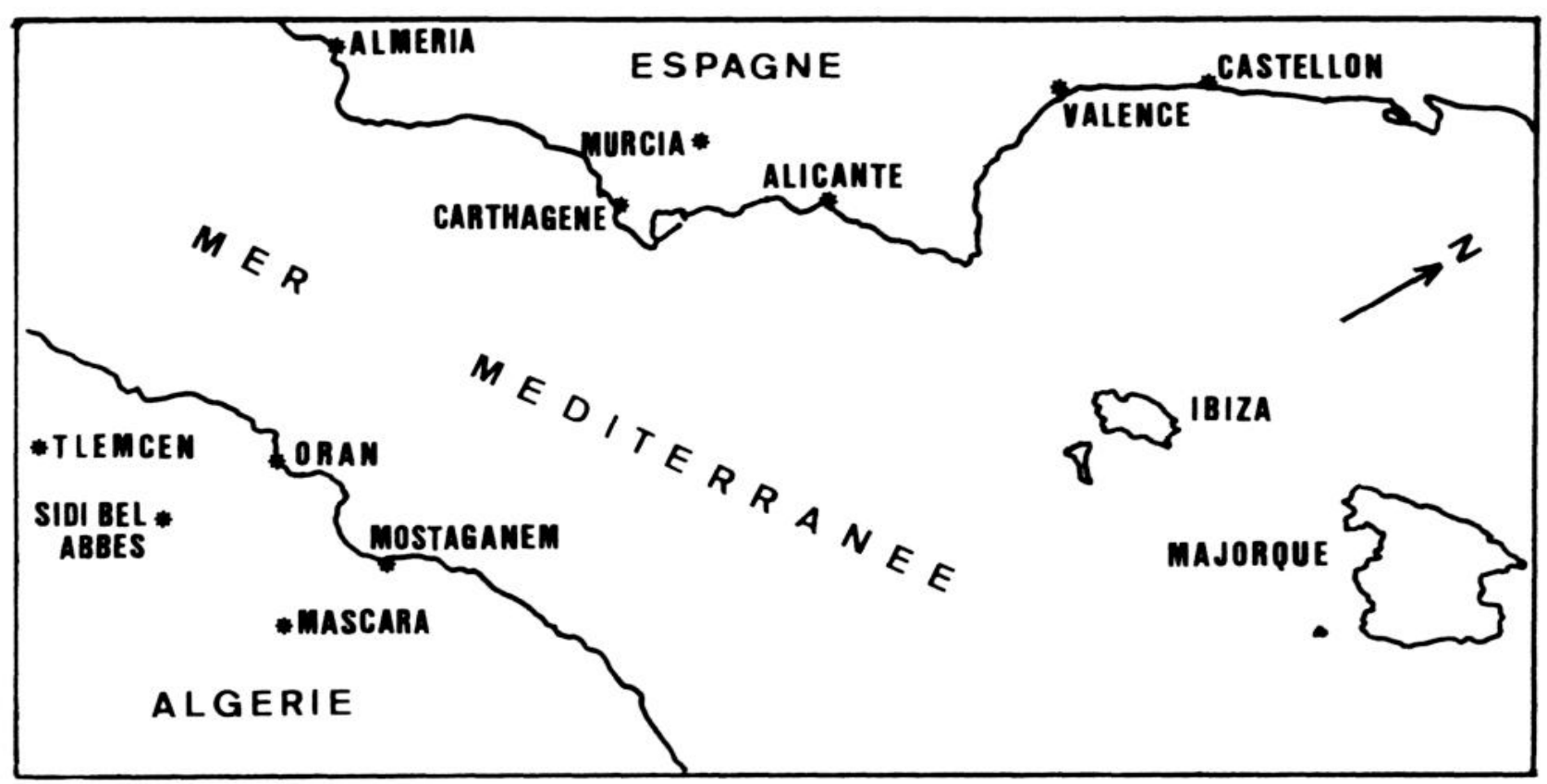

\section{Les origines levantines de la société pied-noire}

Toutefois, la raison fondamentale pour laquelle cette zone a été un centre d'accueil important demeure sans doute l'ascendance espagnole et notamment levantine d'une partie des pieds-noirs du centre et surtout de l'ouest de l'Algérie coloniale, à la fois conséquence et facteur de renforcement de l'influence levantine et alicantine sur l'Oranie.

Longtemps avant la conquête française, la monarchie hispanique a occupé pendant plusieurs siècles la ville d'Oran ${ }^{5}$ ainsi que d'autres places nord-africaines dans le but de maîtriser la Méditerranée occidentale et de surveiller les bases de pirates barbaresques qui ont régulièrement ravagé le sud-est de la péninsule tout le long de l'époque moderne.

5 La ville d'Oran - dont la fondation est attribué à des marins andalous musulmans au début du $X^{c}$ siècle - a été en fait plus longtemps sous domination espagnole (1509-1708 et 1732-1792) que sous l'admnistration turque, française ou algérienne. 
Les relations contemporaines commencent avec la colonisation française de l'Algérie. Depuis la conquête d'Alger en 1830 jusqu'au dernier jour de la Guerre Civile espagnole, l'émigration levantine et baléare vers l'Algérie est très intense, particulièrement vers l'Oranie. Bien avant que les départs vers l'Amérique et les protectorats marocains ne deviennent considérables, l'Algérie a été la destination des émigrés politiques et économiques du sud-est de l'Espagne. L'intensité de ce mouvement migratoire, déjà très important au milieu du XIX" siècle, est étroitement liée aux conflits, aux changements de régime et aux crises économiques qui secouent l'Espagne pendant ces décennies. Dès les débuts de la colonisation française, dans le Levante espagnol on recrute de la main-d'ouvre pour l'agriculture algérienne, dont les salaires ont toujours été plus élevés que ceux de la région (Bonmati 1988: 55). Les facilités d'embauche et les relations familiales entraînent les regroupements qui contribuent à former des chaînes migratoires et à alimenter l'affluence saisonnière, temporaire puis définitive des Espagnols dans la colonie. L'Algérie et le Levant espagnol, plus particulièrement l'Oranie et la région d'Alicante échangent dès le milieu du XIX" siècle des flux importants de travailleurs et de familles et les réseaux de relations économiques et culturelles se renforcent (Simon, 1995 : 16). D'après les recensements, en 1896 il y avait 157560 résidents espagnols en Algérie dont 56000 seraient alicantins (Gozálvez, 1972 : 51). Selon Benjamin Stora (1991: 31), en 1911, l'Oranie comptait 93000 Espagnols et 92000 Espagnols naturalisés français et 95000 autres Français. En 1931 « on estimait la population oranaise originaire d'Espagne à $65 \%$ du total des Européens, 4l \% étant déjà naturalisés » (Stora, 1991 : 60). Tout au long du XIX" siècle l'immigration espagnole en Algérie est si importante que les autorités françaises ont craint la «menace espagnole ». Pour la contrecarrer, elles ont généralisé la scolarisation obligatoire pour tous les enfants d'Européens et ont facilité les naturalisations ; en 1889 une loi attribue automatiquement la nationalité française à tous les Européens nés en Algérie. Cette politique d'assimilation presque forcée explique comment à peine quelques générations après - à la veille de l'indépendance la population européenne non française est devenue si minoritaire.

La phase ultime de ce courant migratoire correspond aux derniers jours de la Guerre Civile espagnole. Alicante fut la dernière ville à tomber - le 30 mars 1939 aux mains des troupes insurgées du Général Franco. Son port fut le dernier théâtre de la guerre, le dernier espoir des soldats républicains. D’après certains auteurs, plus de 15000 personnes s'y concentrèrent dans l'espoir d'être évacuées. Pourtant, ne voulant plus s'engager contre les vainqueurs, le gouvernement français n'accorda pas l'indispensable protection navale qu'il avait pourtant promise et qui aurait permis l'approche de bateaux pour évacuer les réfugiés (Martínez Leal, 1986 : 166). Pendant le mois de mars 1939, six bateaux seulement quittèrent le port emmenant en Algérie environ 5000 réfugiés, le dernier navire - le Stanbrook - partit vers Oran, surchargé de 3 ()28 passagers, le 29 mars 1939.

La rupture politique de 1939 signifie la fin immédiate d'une relation séculaire entre Oran et Alicante ; la Seconde Guerre mondiale et l'isolement international auquel l'Espagne est soumise après la défaite de ses alliés réduisent considérablement les échanges entre les deux régions et empêche l'émigration de reprendre. Mais les 
relations personnelles entre les Espagnols d'Algérie et leurs parents d'Espagne n'en furent pas pour autant coupées ; dans les années cinquante les relations institutionnelles reprennent avec le voyage de plusieurs délégations municipales.

\section{La présence séculaire des Français à Alicante}

La présence et la longue influence de la France dans la province ont joué un rôle important dans l'établissement des Français d'Algérie. En effet, avant le début de la colonisation de l'Afrique du Nord, la région avait déjà établi d'étroites relations avec la France. Alicante a longtemps été, par sa facilité d'accès au centre du pays, le port du Royaume de Castille en Méditerranée et donc un des ports les plus importants d'Espagne. Bien qu'elle ne soit qu'une ville de province cette réalité justifie sa longue vocation cosmopolite et commerciale. Les consulats de plusieurs puissances étrangères y sont présents depuis l'Ancien Régime. Dès le XVIII" siècle on constate l'existence d'un consulat et d'une présence française ; déjà en 1754, forte de 113 hommes de plus de 15 ans, elle constitue la colonie étrangère ${ }^{6}$ la plus importante de la ville, loin devant celle des Génois (Giménez, 1981 : 66). Dans une ville de quelques milliers d'habitants c'est une présence considérable mais irrégulière, très dépendante des traités et des alliances entre les monarchies de France et d'Espagne jusqu'à la fin de l'époque moderne.

La conquête de l'Algérie rapproche à partir de 1830 la France d'Alicante, mais c'est surtout à la fin du XIX' siècle que l'extraordinaire expansion de la viticulture dans la région, après les ravages du phylloxera dans les vignobles français, porte à son apogée la présence française à Alicante. C'est de cette époque que date la première école française de la ville qui comme le Consulat Français, va exister jusqu'à la Guerre Civile espagnole.

Pendant les années 1920, au début de l'aviation commerciale, la course pour l'Atlantique-Sud fait de l'Espagne une plate-forme incontournable des nouvelles lignes marchandes. Alors que les Allemands font étape à Séville, Alicante devient une plaque tournante de la Compagnie Générale Aéropostale, un des précurseurs d'Air France. Pendant les années vingt et trente ses avions et ses pilotes font escale à Alicante tandis que des vols commerciaux relient régulièrement Alicante à Oran et à la France.

Lorsque les pieds-noirs arrivent en 1962 ils retrouvent une présence française à Alicante; celle-ci est en train de reprendre de l'importance grâce aux débuts du tourisme et au différentiel des coûts de la vie qui rendent certains sectcurs comme l'immobilier, très attrayants pour les Français [Petiot de Laluissant, 1970 : 2].

De nos jours la prédominance française se maintient dans la ville; selon le dernier recensement disponible, celui de 1996, les Français demeurent, et de loin, le premier groupe de résidents étrangers dans la municipalité. Par contre à l'échelle

6 Les Français d’Alicante de l’époque travaillaient majoritairement dans les activités commerciales (Giménez, $1981: 67$ ). 
provinciale, avec 4204 personnes, les Français ne sont plus que le cinquième collectif d'étrangers en termes d'importance numérique juste après les Belges, les Hollandais et loin derrière les Allemands et les Britanniques pour qui Alicante est devenue depuis le début des années soixante-dix une des principales destinations touristiques et de retraite?

\section{L’Algérie : une indépendance difficile}

Au-delà de ces explications liées à la proximité géographique et à l'histoire des relations entre les deux espaces, il y a des causes d'ordre conjoncturel qui justifient l'arrivée de pieds-noirs en Espagne. Il s'agit de la dimension politique de l'exil à Alicante des militants de I'OAS (Organisation Armée Secrète), mouvement clandestin qui a tenté de s'opposer par la violence à l'indépendance de l'Algérie entre 1961 et 1963. C'est une question épineuse, fréquemment remise sur le tapis par les médias français. Après le désordre et la confusion de la fin de la guerre, de nombreux activistes de l'Algérie française ont eu des comptes à rendre à la justice ; ne pouvant pas rentrer en France ils ont cherché d'autres régions d'exil. Il est incontestable que l'Espagne et Alicante ont effectivement été le refuge idéal pour les activistes de l'OAS ${ }^{\gamma}$ en attente d'une amnistie qui leur sera finalement accordée en 1968. Le quotidien Información du 24 juillet 1962 signale que les chefs les plus importants de l'OAS : Ortiz et Lagaillarde, leaders des barricades d'Alger de janvier 1961, se sont rendus à Alicante en juillet 1962. Il est également probable que le Général Salan, principal dirigeant de l'OAS, ait séjourné à Alicante après le putsch d'avril 1961, alors qu'il est déjà passé à la clandestinité. Leur passage à Alicante aurait donc été une étape permettant à certains d'entre eux de rester hors d'atteinte de la justice française, pas un seul de ces activistes ne fut extradé par les autorités espagnoles vers la France, pas une seule question ne fut posée sur l'origine des biens avec lesquels certains réfugiés avaient débarqué.

De nos jours la majorité des pieds-noirs qui ont été membres de l'OAS n'ont aucun mal à le reconnaître, mais en dépit des années passées ceux qui en savent le plus se montrent toujours très réticents à parler de la question. Peu d'entre eux nient l'existence passée d'une certaine organisation de l'OAS à Alicante, mais personne ne confirme les informations très contestables que la presse a parfois diffusées en France selon lesquelles il y ait eu près de la ville un camp d'entraînement de l'OAS :

"La police française vient de découvrir l'existence, à Alicante, en Espagne. d'un camp de formation et d'entrainement O.A.S qui s'étend sur 12 fermes. Plusieurs centaines de déserteurs et de Fransais d'Algérie y sont formés militairement pour constituer des commandos spéciaux ayant pour unique mission l'assassinat du Général de Gaulle. "(L'Express, 27 septembre 1962).

7 De ce fait Alicante a longtemps été la première province d'Espagne en nombre d'Européens (Valero, 1992: 11), elle demeure encore de nos jours la première province par le nombre de résidents d'Europe occidentale (Union européenne, Suisse, Norvège et Islande) devant Madrid, Málaga et Barcelone.

8 C'est d'ailleurs à Madrid que l'OAS a été fondé au début de 1961.

REMI 2001 (17) 3 pp. 173-198 
"La réputation d'Alicante justifiait pourtant nos croyances. En 1962, ils araient été jusqu' à cent mille piéds-noirs, en majorité originaires de l'Oranais, à s'installer là dans l'attente de jours meilleurs. Plus de deux mille commandos Delta y campèrent av'ec la bienveillance des autorités franquistes autour des chefs historiques de l'OAS... " (Le Monde, $1^{\mathrm{er}}$ avril 1986).

On peut donc supposer qu'Alicante a été le refuge de nombreux activistes de l'OAS rentrés en France au fur et à mesure des lois d'amnistie, qu'il y a eu une majorité de pieds-noirs sympathisants mais qu'en fait il n'y a eu aucune intention sérieuse d'organiser et de continuer la lutte. D'après les témoignages, les autorités espagnoles ont dû être fermes sur cet aspect : pas d'armes. Ceci est compréhensible, le gouvernement espagnol n'avait, apparemment, aucun intérêt à s'impliquer publiquement dans la lutte de l'OAS en France et celle-ci n'avait aucun intérêt à provoquer les autorités qui lui donnaient réfuge. Le combat s'est surtout réduit à revendiquer l'amnistie et les indemnisations de l'État français. Toutefois, les autorités françaises ont mis du temps à oublier l'attitude permissive de l'Espagne à l'égard de l'OAS'.

\section{De bonnes conditions d'accueil}

La plupart des pieds-noirs d'Alicante signalent qu'ils ont gardé un bon souvenir de la solidarité et de l'accueil chaleureux et convivial reçu des autorités et de la population à leur arrivée en 1962. S'il est vrai que la majorité de la population qui n'avait aucune relation directe avec les pieds-noirs demeura indifférente à leur établissement il n'en est pas moins vrai que la société locale de l'époque, éprouvée par les difficultés d'une guerre civile encore récente, fut particulièrement bienveillante et multiplia les gestes anonymes envers les réfugiés. Les contacts et les connaissances ont joué, dès les premiers instants, un rôle décisif ; même dans les moments les plus difficiles, notamment pendant la nuit du 30 juin 1962 au cours de laquelle plus de 2000 pieds-noirs débarquèrent dans le port, la presse affirme que tout s'est parfaitement déroulé ${ }^{\mid()}$. Il n'y a eu ni mesures de précaution envers ceux qui n'avaient pas de papiers, ni garde à vue pour les ex-combattants de la Guerre Civile espagnole, ni bien évidemment pour les éventuels activistes de l'OAS. Selon les témoignages, quelques heures après le débarquement, tous les nouveaux venus avaient trouvé un logement, apparemment des familles entières n'ayant pas de point de chute ont été hébergées pendant des semaines par des habitants de la ville qui, par ailleurs les ont aidés à trouver du travail. Malgré la mobilisation de l'administration et de la Croix Rouge on ne retrouve aucune trace — dans les archives municipales — d'un déblocage de fonds publics pour faire face à la présence de plusieurs centaines de réfugiés. Plus

9 Plusieurs personnes ont d'ailleurs comparé ce comportement du gouvernement espagnol avec celui du gouvernement français à l'égard des membres de l'ETA réfugiés en France pendant les années 1970 et 1980.

10 Selon les titres du quotidien Información du lendemain : « 2200 Espagnols arrivés d’Oran. Des vivats à Franco et à l'Espagne dans les instants, plein d'émotion, de fouler la terre espagnole ». 
tard, les banques et les caisses d'épargne accordent avec grande facilité des prêts, sans caution et à bas intérêt, à l'initiative - selon les rumeurs de l'époque — du Général Franco lui-même (Palacio, $1968: 45$ ). Les autorités et quelques immigrés ont rapidement compris le rôle de catalyseur que pouvaient jouer les pieds-noirs dans le développement de la région.

Toutes ces données doivent, bien évidemment, être confrontés à l'accueil que les autorités oranaises ont donné aux vaincus de la Guerre Civile espagnole en 1939 et avec l'impression que les pieds-noirs ont gardé de leur arrivée en métropole et des difficultés réelles que la France a dû surmonter pour faire face à l'arrivée et à l'adaptation de plusieurs centaines de milliers de rapatriés. Bien avant la fin de la Guerre d'Algérie les autorités françaises s'étaient efforcé d'échelonner l'affluence des réfugiés en multipliant les obstacles" ${ }^{1}$. Marseille est la première ville d'arrivée des rapatriés, elle en a vu passer les deux tiers. Dans une ambiance chaotique, décrite par Jean Jacques Jordi dans son livre 1962: l'arrivée des pieds-noirs, cette ville et ses habitants ont littéralement été débordés par cet afflux pendant l'été 1962. Les métropolitains ont gardé en général un comportement de froideur et de rejet systématique envers les rapatriés, qu'ils voyaient à travers le stéréotype du colon raciste, inculte et borné qui se répandait à Marseille (Jordi, 1995: 49). Les pieds-noirs, quant à eux, se sentaient haïs et incompris par la France qui les avait abandonnés en Algérie. Ceux qui n'ont en France aucune relation familiale sont distribués dans l'ensemble du pays, mais beaucoup d'entre eux, envoyés dans les régions septentrionales, déçus et inadaptés reviennent dans le Sud attirés par la proximité de l'Algérie. Certains, influencés par des amis, des parents ou par les nouvelles des piedsnoirs à Alicante, décident de les rejoindre.

Les raisons que nous venons d'examiner expliquent pourquoi plusieurs milliers de Français d'Algérie ont choisi Alicante pour refaire leurs vies. Leur ascendance espagnole, l'accueil et les rapports qu'entretiennent ces deux régions sont des facteurs qui justifient cet établissement en Espagne et le « bonheur » d'y vivre que les médias se sont chargés de transmettre tout le long des trente années suivantes. Néanmoins, cette réalité ne doit pas cacher qu'une grande partie des pieds-noirs d'origine espagnole ne se sont pas établis en Espagne, mais en France. Malgré l'émigration séculaire des levantins en Algérie et l'importance de la composante espagnole dans la société européenne de l'Oranie, la grande majorité de la population espagnole ou d'origine espagnole d'Algérie a participé au grand flux des rapatriés vers la métropole ${ }^{12}$. L'Espagne franquiste demeure pour la plupart d'entre eux un pays

11 Les formalités administratives se multiplièrent et en janvier 1962 le gouvernement réduisit à trois le nombre de liaisons entre la France et l'Algérie. Le 16 mai 1962, face à l'affluence, ce sont les compagnies qui décidèrent unilatéralement de réaugmenter le nombre de traversées (Jordi, $1995: 19)$.

12 On ne les a d'ailleurs pas retrouvé, d'après Guy Hermet (1968:38) les Espagnols d'Algérie arrivés en France en 1962 ne sont que 6 500. Aucune statistique ne permet d'évaluer le nombre, sans doute beaucoup plus important, des Français d'Algérie d'ascendance espagnole immigrés en France. Seuls les noms - très souvent des noms espagnols accolés à un prénom français - indiquent l'origine espagnole d'une grande partie des pieds-noirs installés en métropole. 
difficile à cause du passé, de son régime politique et de son ouverture internationale et économique encore limitée.

\section{LES EFFETS SUR UNE VILLE EN PLEINE EXPANSION}

Depuis la fïn de la Guerre Civile, Alicante était devenue une petite capitale de province éloignée des pôles de développement promus par l'État. Vingt-trois ans après la fin du conflit la population progresse lentement dépassant à peine 100000 habitants. Après ces années d'isolement international, la ville a renoncé au cosmopolitisme méditerranéen qui avait été sa caractéristique depuis le début de l’âge moderne. Mais l'ouverture économique et touristique amorcée par l'Espagne dès la fin des années cinquante $^{1.3}$ s'est faite surtout sentir dans les provinces du littoral méditerranéen. Pendant la décennie suivante Alicante reçoit chaque année plusicurs milliers d’immigrés internes, originaires pour la plupart de Murcie, de Castille et d'Andalousie : la région offre en effet d'exceptionnelles perspectives pour les affaires et la spéculation foncière. C'est donc au début d'une époque de changements, de mutations sociales et de décollage économique que des milliers de Français d'Algérie se sont installés dans la zone contribuant de ce fait très activement à l'évolution de la région.

\section{L’apport démographique : la question des trente mille pieds-noirs}

Trente mille est le chiffre rond cité le plus souvent pour évaluer le nombre des pieds-noirs qui se sont installés à Alicante ; c'est le constat qui, dès le début, est adopté par les médias, la société et les différentes études réalisées dans la région. Il est impossible de connaître avec certitude la source statistique qui a donné lieu à cette évaluation ; l'origine de sa diffusion est certainement à rechercher auprès des piedsnoirs eux-mêmes et à la revue hebdomadaire qu'un groupe d'entre eux commence à éditer en 1965 : Le Courier du Soleil. Dès son premier numéro cette publication fait couramment allusion aux trente mille pieds-noirs, sans jamais préciser sa source.

Par ailleurs, en 1968 Antoni Seva Llinares publie Alacant : trenta mil piedsnoirs, seul ouvrage sur le phénomène pied-noir à Alicante. Cette référence est souvent citéc dans les recherches el dans les auvres qui abordent l'évolution sociale, économique ou démographique de la ville et de sa province. Elle contribue aussi à l'acceptation de l’évaluation. même si l'auteur lui-même, précise, sans citer ses sources, qu'il y aurait 28000 pieds-noirs dans la ville et 12000 dans le reste de la province (Seva Llinares, $1968: 113$ ).

13 Leéchec des politiques autarciques prônées à la suite de la Guerre Civile mènera au cours des années 1950 a une lente libéralisation économique qui aboutira. le 21 juillet 1959. à la promulgation de la «Loi de Nouvel Aménagement Économique " avec le soutien du Fonds Monétaire Internationale. Cette loi connue sous le nom de «Plan de Stabilisation " marque le début de la modernisation de l'économie dont certaines conséquences sont l'entrée de capitaux étrangers, lapparition du chômage, la croissance des différences sociales. l’exode rural et l'émigration internationale. Ce sont les bases du décollage économique espagnol qui durera jusqu à la fin des années soixante-dix. 
Ce nombre et cette imprécision dans les sources sont certainement volontaires. Les pieds-noirs avaient tout intérêt à gonfler numériquement leur colonie afin de signifier au gouvernement français qu'il avait un problème à Alicante. En réalité, il s'agit d'un nombre bel et bien excessif pour une ville où 121823 personnes sont recensées en 1960. L'établissement de trente mille immigrés aurait représenté une augmentation du quart de la population en quelques mois ce qui est invraisemblable selon les témoignages. La croissance démographique est très forte à cette époque (une moyenne de six mille personnes par année entre les recensements de 1960 et de 1970), mais pas assez pour y voir l'arrivée de 30000 résidents en plus de l'accroissement naturel et l'exode rural très importants de ces années. Nous savons en effet que les étrangers représentent moins du dixième de l'immigration totale (Quiñonero Fernández, $1988: 183$ ).

Déterminer l'ordre de grandeur du collectif pied-noir installé à Alicante à la suite de l'indépendance de l'Algérie est pourtant essentiel pour connaître leur influence dans la ville, mais la question n'est pas facile. Dès le début, statistiques, évaluations personnelles et médiatiques sont divergentes, voire contradictoires. Au cours des années la presse donne des approximations qui varient généralement entre 20000 et 45000 personnes. Mais pas un seul article ne cite ses sources et n'apporte de données concrètes ; ils restent flous en ce qui concerne l'espace (ville, province...) et la population (Français, Espagnols, d'Algérie, du Maroc...) à laquelle ils font référence. De leur côté les statistiques disponibles donnent, presque toutes, des résultats complètement différents, mais qui ne nous rapprochent pas davantage du nombre réel de Français d'Algérie à Alicante. D'après ces sources quelque deux à trois mille personnes - françaises ou espagnoles - seraient venues d'Algérie, ce chiffre est bien au dessous des trente mille personnes recherchées et pourtant citées dans bon nombre de publications ${ }^{14}$.

Le nombre et l'estimation les plus vraisemblables proviennent du Consulat de France à Alicante réouvert en 1967, précisément à la suite de l'installation des piedsnoirs dans la région. En 1970, dans un rapport à l'Ambassadeur de France à Madrid, le Consul Adjoint à Alicante, Monsieur Petiot de Laluisant, affirme que " six mille Français, majoritairement d'Algérie, sont immatriculés au Consulat d'Alicante ", mais il estime de dix à quinze mille le nombre de Français qui vivent dans la province.

Nous avons donc la certitude que dès 1962 il y a eu à Alicante des registres avec au moins six mille Français, certainement d'Algérie, mais ces données n'ont pas été retrouvées. Les statistiques espagnoles, sans doute moins exhaustives, permettent cependant de cerner les évaluations à partir des chiffres officiels et surtout d'observer

14 D'après le dernier recensement (1996) la province d'Alicante compte 1369708 habitants et la capitale 274 577. Dans la municipalité il y a 1008 résidents nés en Algérie et 2367 nés au Maroc, 210 ont la nationalité algérienne et 280 la nationalité marocaine. Ces habitants nés en Afrique du Nord mais qui non pas une nationalité maghrébine sont des immigrés de la décolonisation étant donné que les maghrébins naturalisés étaient encore peu nombreux à cette époque. 
les principales caractéristiques sociales et démographiques de cette population. La meilleure source accessible pour obtenir des informations détaillées sur les pieds-noirs est le Padrón Municipal de Habitantes de 1965 (le registre municipal de population établi en 1965). Il s'agit du premier dénombrement de population fait après l'indépendance de l'Algérie. À partir de ce Padrón nous avons isolé les personnes originaires d'Afrique du Nord. Nous avons donc considéré comme tels les personnes qui sont nées en Algérie ou au Maroc ${ }^{15}$ et les familles dont la plupart des membres y sont nés, ainsi que celles qui ont déclaré vivre dans un des deux pays en 1960, date du recensement antérieur.

Tableau 1 : Dénombrement des personnes originaires d'Afrique du Nord recensées à Alicante en 1965

\begin{tabular}{|l|c|c|}
\hline Origine des immigrés & Nombre de familles & Nombre de personnes \\
\hline Algérie & 896 & 2778 \\
Avant 1960 & 393 & 1398 \\
Après 1960 & 503 & 1380 \\
\hline Maroc français & 89 & 323 \\
Avant 1956 & 20 & 68 \\
Après 1956 & 69 & 255 \\
\hline Maroc espagnol & 405 & 1602 \\
Avant 1956 & 137 & 548 \\
Après 1956 & 268 & 1054 \\
\hline Total & 1390 & 4703 \\
\hline
\end{tabular}

Source : Padrón Municipal de Habitantes de 1965, dénombrement personnel

Les résultats du recensement (Padrón) de 1965 sont du même ordre que ceux des autres statistiques officielles; ils restent bien loin des trente mille pieds-noirs présumés et même des « six mille Français, majoritairement d'Algérie » immatriculés au Consulat en 1970. On ne dénombre que 4703 personnes immigrés d'Afrique du Nord dont seule une très petite minorité déclare explicitement (dans la colonne attribuée à la nationalité) être de nationalité française. Parmi elles, 2689 (57\%) seulement seraient venues après la décolonisation, $1309 \mathrm{du}$ Maroc après l'indépendance en 1956 - et 1380 d'Algérie à partir de 1960, date où ont commencé les départs des pieds-noirs.

La différence entre les données recueillies et le chiffre attendu s'expliquent en grande partie par de possibles omissions ou erreurs dans la transcription manuelle des registres de population. Par ailleurs, il semble certain qu'un nombre considérable de

15 Bien que le terme pied-noir ne soit pas très clairement défini et se réfère exclusivement aux Français d'Algérie, il faut dire qu'en Espagne ce nom s'est généralisé aux nombreux Européens anciens résidents du protectorat français au Maroc. Malgré leurs différences avec les anciens résidents d'Algérie, la plupart de ces derniers se considèrent eux-mêmes comme des pieds-noirs. C'est pourquoi nous avons inclus dans notre analyse statistique les anciens résidents des protectorats marocains. 
pieds-noirs ne se sont pas fait recenser ; la plupart de ceux que j'ai interviewé déclarent en effet ne pas l'avoir fait pendant longtemps pour des raisons diverses ${ }^{16}$ : oubli, manque de motivation ou d'information, absence de pression de la part des autorités ou encore espoir de recevoir les indemnisations de l'État français dont bénéficiaient uniquement les rapatriés qui résidaient en France.

D'après toutes les informations obtenues nous pouvons donc conclure qu'il est plausible qu'un nombre important de réfugiés, trente mille, voire plus. soient passés dans la région : il est également probable que beaucoup d'entre eux se sont dispersés dans les villages de la province où ils avaient des parents. Mais il est impensable qu'un tel nombre se soit installé dans la province et encore moins dans la ville. Toutes les sources fiables nous amènent à un chiffre bien inféricur qui serait, pour la deuxième partie des années 1960, de quelques milliers définitivement installés et recensés dans la région. Il est probable que la majorité des immigrés ne se soient pas soumis au recensement à l'époque et qu'ils soient en réalité bien plus nombreux à vivre à Alicante, au plus dix mille dans la capitale. Mais ils ne sont certainement pas dix fois plus que les 2778 immigrés d'Algérie dénombrés dans le registre municipal de 1965.

\section{Une population socialement équilibrée et structurée}

La présence pied-noire à Alicante est avant tout le produit d'une expulsion, d'un exil qui a concerné tous les âges, toutes les classes et tout les secteurs économiques d'une société colonisatrice, certes. mais stabilisée et consolidée, contrairement aux sociétés européennes des autres colonies d'Afrique. Il s'agit d'une population déplacée dont les caractéristiques sociales difèrent des migrations de travail courantes dans les années soixante. Chez les pieds-noirs d'Alicante on ne retrouve pas une majorité d'hommes seuls, jeunes et actifs qui travaillent surtout dans l'industrie ; on a au contraire une migration familiale où la population inactive prédomine largement.

En dépit de l'irrégularité de la pyramide des âges (fig. 3) construite à partir d'une population trop peu nombreuse, on observe qu'il s'agit d'un groupe assez équilibré du point de vue démographique. D’une part nous avons une légère majorité de femmes, $53 \%$ du total (alors que cette proportion n'est que de $46 \%$ pour l'ensemble des immigrés et de $52 \%$, pour toute la population d'Alicante) ${ }^{17}$. De même leur structure par âges rapproche davantage les pieds-noirs de la population autochtone que de la population d’immigrés : $11 \%$ des pieds-noirs ont 65 ans el plus $(9 \%$ des

16 Ne pas être recensé pour une ou plusieurs de ces raisons a longtemps été une caractéristique courante chez les Européens d’Alicante, travailleurs ou retraités. Ce n’est qu à partir de 1974. avec le début de la crise économique et l'augmentation du chômage que l'on commence à exiger et à restreindre sérieusement la concession des permis de travail aux étrangers | Valero. 1992:781

17 Les résultats pour l'ensemble des immigrés sont de Quiñonero Fernández (1988) alors que ceux de la populatoin d'Alicante sont issus des publications du recensement de 1970. 
Figure 3 : Pyramide des âges de la population originaire d'Algérie recensée à Alicante en 1965

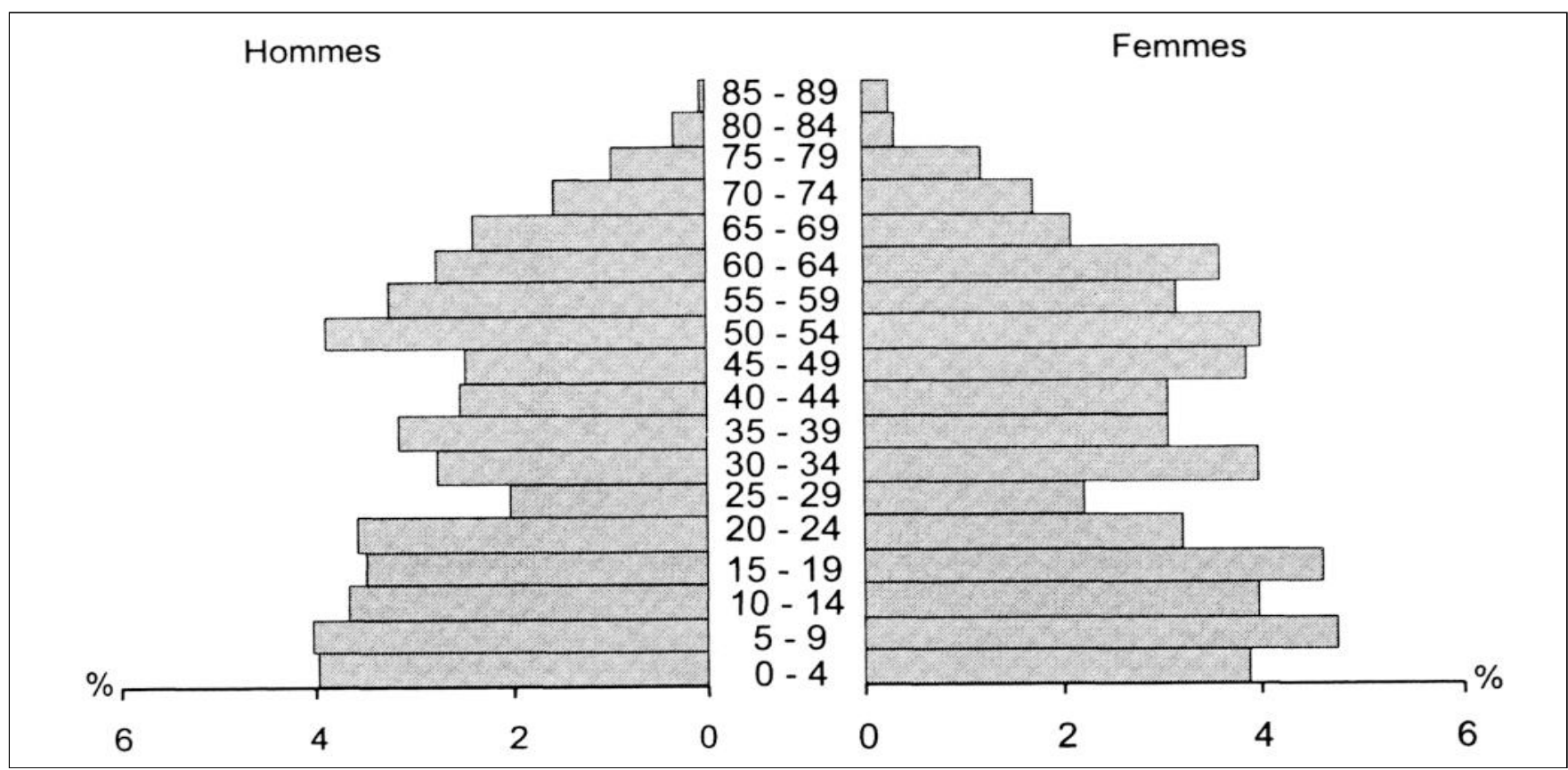

Total : 2778 personnes.

Source : Padión Municipal de Habirantes de 1965, dénombrement et réalisation personnels

autochtones) alors que $24 \%$ d'entre eux ont moins de 15 ans (29\% des autochtones et près de $11 \%$ pour l'ensemble des immigrés).

La principale anomalie de la pyramide est le rétrécissement des classes d'âge centrales, celles des 20-50 ans. Ce resserrement correspond aux tranches d'âge les plus actives : s'agit-il de personnes qui après avoir passé les premiers mois d'exil à Alicante sont reparties étudier ou travailler en France où elles avaient plus de possibilités de continuer à exercer leur métier?

Les inactifs représentent $70 \%$ de la population pied-noire de Alicante ; c'est une proportion forte, qui dépasse celle de la population locale - environ $67 \%$ d'inactifs en 1970 - et à plus forte raison celle de la population immigrée - $58 \%$ en 1981 (Quiñonero Fernández, 1988 : 158). Près de la moitié d'entre eux se déclarent "sans profession" (la plupart des femmes sont dans ce cas). Enfants et étudiants représentent $38 \%$ des inactifs recensés, $14 \%$ sont des retraités, des rentiers et des invalides.

La population active des Européens d'Algérie est représentée dans tous les grands secteurs d'activité (tableau 2). Cependant la majorité d'entre eux travaillent dans le secteur tertiaire (presque deux tiers des emplois) et notamment plus d'un quart d'entre eux déclarent une activité liée au commerce. Il s'agit, comme nous le verrons par la suite, des propriétaires et des employés des boutiques et magasins que les exilés ont créé partout dans la ville bouleversant ainsi son panorama commercial. 
Tableau 2 : Principales activités des pieds-noirs d'Alicante

\begin{tabular}{|c|c|c|}
\hline Secteur d'activité & Nombre & $\%$ des actifs \\
\hline Primaire & 50 & 6,0 \\
\hline Artisanat et industrie & 123 & 14,7 \\
\hline Bâtiment et travaux publics & 85 & 10,1 \\
\hline Tertiaire & 541 & 64,6 \\
\hline Dont commerce & 227 & 27,1 \\
\hline Dont hotellerie/restauration & 28 & 3,3 \\
\hline Dont administration & 31 & 3,7 \\
\hline Quaternaire & 17 & 2,0 \\
\hline Non classés & 22 & 2,6 \\
\hline Ensemble des actifs & 838 & 100,0 \\
\hline
\end{tabular}

Source : Padrón Municipal de Habitantes de 1965, dénombrement personnel

\section{Une petite révolution économique}

Dès la fin des années cinquante la ville commence à bénéficier de la revitalisation sociale et économique qui suit l'ouverture de l'Espagne. Au cours de ces années, son aspect se transforme radicalement : pour loger les milliers d'immigrants qui arrivent tous les ans, de nouveaux quartiers se construisent rapidement autour du centre-ville ; l'espace urbanisé s'étend alors que le grand tourisme fait son apparition, révolutionnant services, commerces et transports. C'est à cette époque et dans ce contexte prometteur, au début de la modernisation économique du pays, que sont arrivés les pieds-noirs. Certains d'entre eux amènent les habitudes, la détermination et la capacité de travail qui caractérisent souvent le monde colonial. Une fois installés, ils trouvent de la part des autorités et de la population les facilités et le laisser-faire qui leur permettront de provoquer une véritable révolution dans certains secteurs de l'économie locale.

La métamorphose urbaine a concerné d'abord l'immobilier, un des secteurs les plus dynamiques de l'économie, un de ceux qui permettent les profits les plus importants ${ }^{18}$. Avec la collaboration de certains investisseurs et la passivité douteuse des autorités, quelques agences immobilières, dont certaines sont très liées à des piedsnoirs, impulsent la construction et la vente de centaines d'appartements.

Un quartier en est devenu le symbole : l'Albufereta. Un complexe touristique et résidentiel, aujourd'hui mal vieilli, mais présenté à l'époque comme l'expression de la modernité, du développement et de la qualité de vie. Deux kilomètres au nord du centre-ville, d'énormes immeubles, certains comptant plusieurs dizaines d'étages, ont été construits en forme de muraille autour d'une petite baie après avoir fait sauté à la dynamite une partie de la montagne qui l'entourait.

18 Au cours de la décennie soixante le nombre de logements de la ville a plus que doublé passant de 33979 à 78507 . 
Figure 4 : Vue actuelle de la Albufereta

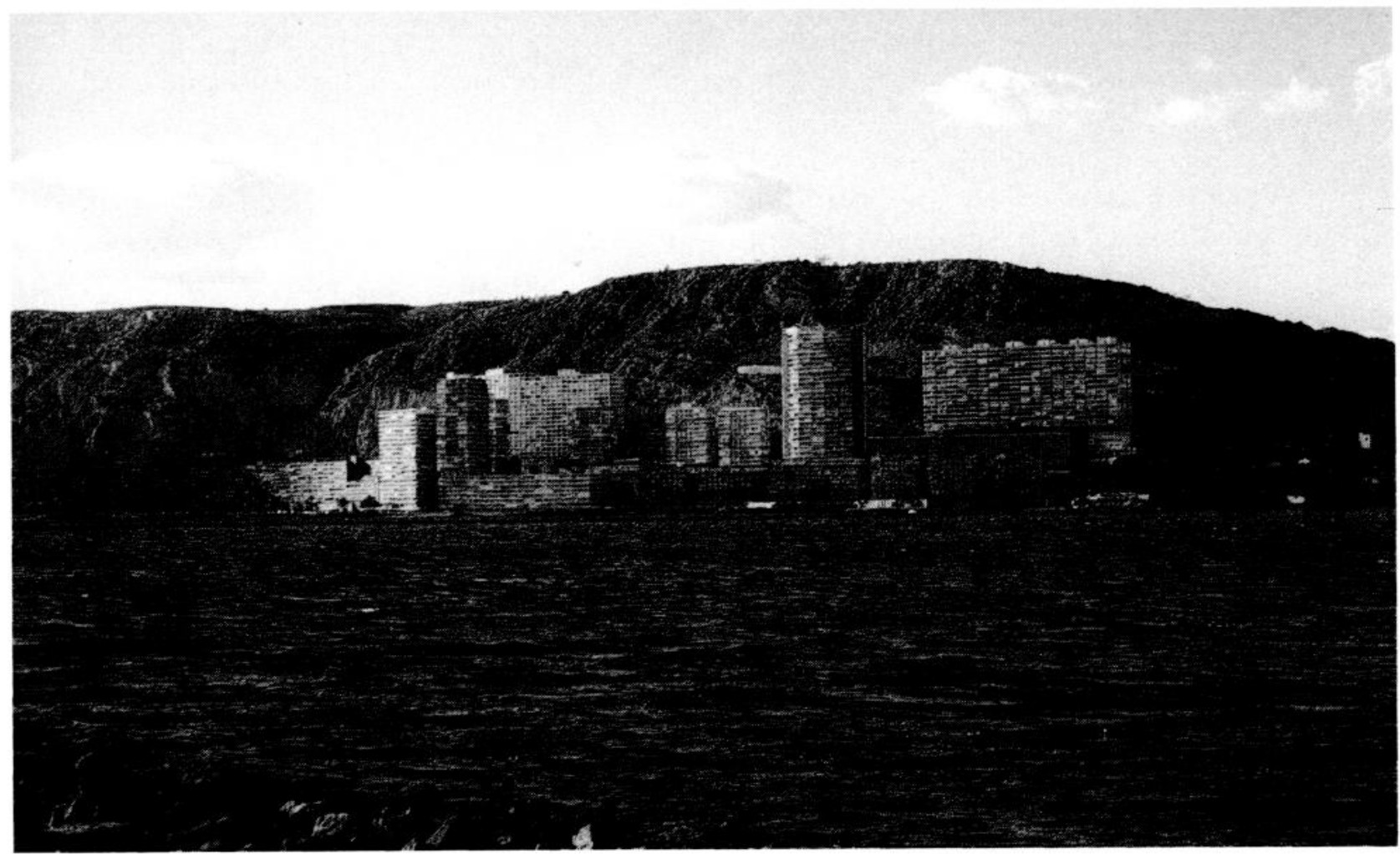

Un des pôles de la ville que quelques promoteurs, privés et publics, voulaient créer tout le long des côtes de la province. D'après les annonces du Courier du Soleil il avait été aussi prévu de construire un quartier semblable sur les hauteurs ; on aperçoit la route gravissant la montagne qui devait y mener.

(Photo : JD Sempere Souvannavong)

Curieusement, le registre de population de 1965 ne révèle que la présence de 27 immigrés d'Algérie dans cette zone ; c'est pourtant dans ce quartier, dont la naissance même est liée à leur arrivée, que les pieds-noirs ont eu le plus d'influence. Aujourd'hui encore, il n'est pas rare d'y entendre parler français dans la rue et dans certains magasins. C'est dans cette zone que se trouve encore le Lycée Français d'Alicante et dans un de ses gratte-ciel qu'au cours de l'été 1967 s'installa la première représentation diplomatique française depuis la Guerre Civile.

Cependant le secteur immobilier n'a été l'affaire que de quelques immigrés. Les pieds-noirs se sont insérés dans presque tous les secteurs de l'économie locale, surtout dans les services et plus particulièrement dans le commerce où un bon nombre d'entre eux ont trouvé un créneau à prendre. Quelques temps après leur arrivée les immigrés commencèrent en effet à ouvrir de nombreuses boutiques de tout genre envahissant les artères principales de la ville. Par la qualité de leurs produits et la nouveauté de leur service, les commerces pieds-noirs ont vite effacé la concurrence locale : des boulangeries, des librairies, des hôtels, des supermarchés, des pressings - jusqu'à ce moment inconnus —, des pâtisseries-confiseries — dont certaines 
existent encore - et surtout des bars, des pubs, des boîtes de nuits, des cafétérias et des restaurants. C'est dans ce domaine, celui des loisirs et de la restauration, qu'ils ont le plus participé à l'évolution économique de la ville et de la région. Plusieurs dizaines d'établissements créés par eux ont marqué la vie urbaine d'une époque et d'une génération.

Même si pendant les premières années les pieds-noirs ont eu une influence indéniable dans la croissance démographique de la ville, leur présence numérique se noie malgré leur nombre dans la grande affluence migratoire des années 1960 et 1970. Certes, il s'agit d'un apport minoritaire, semblable à la population autochtone du point de vue démographique, mais un apport qualitatif capital : entrepreneurs, actifs et dynamiques, les pieds-noirs ont innové dans de nombreux secteurs de l'économie ; leur rôle clef est tout à fait comparable à celui des immigrés nationaux qui ont fourni de la main-d'ouvre au cours de ces années de forte expansion.

\section{UNE INTÉGRATION SOCIALE VARIÉE ET PARFOIS INACHEVÉE}

Les pieds-noirs ont joué un rôle très important sur le plan social, une bonne partie de la société locale s'est laissée entraîner par la façon de vivre des nouveaux arrivants. Symbole de modernité et d'ouverture dans une Espagne encore traditionnelle mais avide d'influences extérieures ${ }^{19}$ ces milliers de Français ont été adoptés sans difficultés, « sans se rendre compte, sans indigestions, la ville ingérait une bonne dose de corps étrangers » (Seva Llinares, 1968: 119). Leur installation n'a pas créé de problèmes majeurs ce qui serait impensable de nos jours ; néanmoins pour différentes raisons certains d'entre eux $n$ 'ont jamais réussi à s'intégrer dans la société et sont restés assez imperméables à leur nouvel entourage.

\section{«... farouchement Français, plus cocardiers que jamais. »}

Malgré le ressentiment que les pieds-noirs avaient à l'égard du gouvernement de la métropole ils sont restés pour la plupart très attachés à leur identité française ${ }^{20}$; selon les mots de Léo Palacio "...farouchement Français, plus cocardiers que jamais» (1968: 50). Comme en Algérie, leur nationalisme reste un patriotisme de frontière, de résident à l'étranger qui n'a aucune volonté de se fondre dans la société locale. De nos jours encore beaucoup de pieds-noirs parlent surtout en français (le français pied-noir) et lorsqu'il s'exprime en espagnol ils conservent un fort accent.

19 «... beaucoup (d'alicantins) se demandent encore comment diable ils ont pu abandonner. Angelita, leur fiancée de toujours, pour draguer avec Janine ; comment les filles de Jesús y María (l'école B.C.B.G d'Alicante) perdirent du jour au lendemain tout leur charme et se convertirent en un rêve fade et sans couleurs d'une vielle photographie. " (Harpo, 1985).

20 Selon le premier Consul-adjoint, Monsieur Petiot de Laluisant, huit ans après l'indépendance "une seule famille a demandé (par rancoeur) sa naturalisation espagnole " (Petiot de Laluisant, $1970: 22$ ). Ce n'est pas exactement ce qu'a dit Agatangelo Soler, Maire d'Alicante en 1962 : "la première chose qu'ils faisaient c'était aller à la Mairie pour déchirer leur passeport » (Infornación, 2 février 1992). 
Signalons ainsi comme particularité le vote des Français de la région : certes, leur participation aux scrutins est faible par rapport à la métropole (moins du $20 \%$ des inscrits sur les listes électorales du Consulats Général), mais en 1994, le Front National obtient dans la circonscription du Consulat d'Alicante des résultats remarquables que l'on ne retrouve pratiquement nulle part ailleurs et qui font de cette ville espagnole un bastion du parti nationaliste et populiste français. Lors des élections au Parlement Européen de cette même année, alors que dans les consulats généraux de Barcelone et de Bilbao, la liste menée par Le Pen obtenait $6 \%$ et $3 \%$ des votes respectivement, à Alicante il prenait aisément la première place avec $32 \%$ des voix face aux candidats de droite et de gauche qui n'en obtenaient que $20 \%$ et $17 \%$. Quant aux élections au Conseil Supérieur des Français à l'Étranger, le Consulat d'Alicante représentait lui seul presque la moitié des 703 votes obtenus par la liste du Front National pour toute l'Espagne. En 1999, même si le Front National, divisé, perd sa primauté, il conserve un pourcentage de vote largement supérieur à celui de la métropole ${ }^{21}$. Cette réalité révèle bien une spécificité des Français d'Alicante ; est-elle due au vote pied-noir?

Les conditions dans lesquelles ils sont partis aident à comprendre ce nationalisme des Français d'Algérie : l'exil représente un déracinement brusque et violent, un traumatisme d'autant plus fort qu'ils ont la certitude absolue qu'aucun retour n'est envisageable. Malgré la proximité de leur pays natal, la grande majorité de pieds-noirs n'y sont jamais retourné et souhaitent rarement le faire : c'est un monde révolu. Cette réalité, mêlée à la forte ressemblance de la région d'Alicante et de leur environnement en Algérie a contribué à entretenir pour toute cette génération le culte du passé et le mythe du paradis perdu ; une dimension à retenir pour comprendre pourquoi, pendant ces années de très forte immigration, ils ne se sont pas trop mélangés à cette société hétérogène. Par rapport aux immigrés nationaux, ils ont une origine différente et commune ; en dépit des éternelles rivalités entre les régions de l'Algérie coloniale un sentiment d'unité, forgé dans ces circonstances difficiles, leur a donné une identité propre et solide ainsi qu'une certaine discipline de groupe ${ }^{22}$. Par l'importance suffisante de leur nombre, leur vitalité et le laissez-faire des autorités ${ }^{23}$ les pieds-noirs d'Alicante ont créé un monde à eux qui, même s'il a joué un rôle dans la vie locale les a maintenu un peu à l'écart du reste de la société.

21 Lors des élections au Parlement Européen du 13 juin 1999 la participation n’atteignit pas $7 \%$ des 4762 inscrits dans les listes électorales du Consulat. La liste conduite par Le Pen fut. après celles du PS $(27 \%)$ et du RPR $(16 \%)$ la troisième avec $15 \%$ des votes. La liste conduite par Megret fut la sixième avec $8 \%$ des voix el celle de Pasqua cinquième avec $9 \%$.

22 Nous nous référons entre autres aux services professionnels, les pieds-noirs préfèrent aller chez des professionnels pieds-noirs (médecins, avocats ...) même s’ils doivent faire de longs déplacements.

23 Un " laissez-faire " comparé à la situation en France où face à l'affluence de rapatriés la politique a clairement été celle d'éviter qu'ils se concentrent dans certaines régions et qu ils s'organisent trop. Un Ministère des Rapatriés est mis en place à la fin de 1962 pour s'occuper de leur intégration et de leurs indemnisations, il disparaîtra rapidement. dès 1964 (Dubois. $1993: 106)$. 


\section{Initiatives et formes d'organisation dans l'exil}

Dans ce contexte, les pieds-noirs ont tenté de mettre en place toute une infrastructure destinée à conserver et à préserver leur identité française et pied-noir dans un pays qui n'était ni la France ni l'Algérie. Comme en métropole, au cours des années d'exil, plusieurs organisations à vocation politique et associations amicales ont été fondées avec plus ou moins de succès par les pieds-noirs d'Alicante pour défendre leurs intérêts en France. L'amnistie a été le but essentiel des ces organisations : des négociations entre le gouvernement français et les représentants des pieds-noirs d'Alicante ont eu lieu quelques temps après leur arrivée ; rappelons aussi le thème controversé des indemnisations auxquelles seuls les résidents en France ont droit.

Il y a eu des tentatives de créer une seule et unique association pour l'ensemble des anciens d'Afrique du Nord (Maroc et Algérie), mais faute d'objectifs communs ces projets ont échoué en dépit des aspirations de bon nombre d'entre eux. Trop différents par leur vécu les anciens résidents du Maroc (où le conflit de l'indépendance a été beaucoup moins violent) et ceux d'Algérie ne se sont jamais entendu sur le plan organisationnel. Cette incompatibilité est claire dans les types d'associations créées; alors que les anciens du Maroc ont un club de pétanque où ils se réunissent pour jouer et pour être ensemble, les anciens d'Algérie sont à l'origine de plusieurs associations dont le but est de protéger leur identité et d'entretenir le souvenir: l'Association des Anciens d'Afrique du Nord, l'Association des Anciens Combattants, l'Association de Bienfaisance aux Français, le Cercle Algérianiste et l'Union des Français. Certaines de ces associations ont disparu essentiellement à cause du manque de motivation des jeunes beaucoup plus intégrés dans la société locale; de nos jours c'est souvent l'aspect des amicales qui prévaut ; comme en France les piedsnoirs organisent régulièrement des rencontres de convivialité : repas, méchouis, excursions et rallyes ou matches de football.

D'autres initiatives sont prises, Le Courier du Soleil déjà cité mérite une mention spéciale : avec un tirage de 15000 exemplaires distribués dans toute la province, en Espagne et en France (Seva Llinares, 1968: 131) cette revue hebdomadaire éditée à l'instar d'autres publications pieds-noirs en France est un véritable tour de force. Elle prouve la ferme volonté d'un groupe de maintenir une cohésion entre les Anciens d'Afrique du Nord et de montrer leur intégration dans la société alicantine. Dans la rubrique "L'ami de la semaine » on peut lire des entretiens avec des personnalités choisies du monde pied-noir comme le boxeur Max Michel, ou Pierre García « el emigrante », le torero algérois (victime d'un coup de corne lors de sa première sortie dans l'arène en mai 1966). Le but de cette rubrique et de la publication en général était de montrer comment les pieds-noirs se sont parfaitement intégrés dans leur nouvelle vie. Mais à aucun moment la revue ne profite de sa liberté d'action pour essayer de capter des lecteurs espagnols qui auraient pu s'intéresser à une publication plus libre et plus critique que ne l'étaient les journaux de l'époque encadrés par le régime et dénués d'intérêt. De sa naissance à sa disparition, Le Courier du Soleil est la revue des Français d'Algérie pour les Français d'Algérie ; ses articles encensent les charmes de la vie à Alicante, sa gastronomie, son tourisme, l'univers pied-noir et ils abordent aussi de manière subjective des aspects politiques. Malgré la volonté des débuts de maintenir la revue à l'écart du débat politique, peu à peu les articles de fond 
prennent le dessus et gagnent en virulence, ils critiquent le Général de Gaulle et font l'apologie du régime franquiste, notamment lors du référendum organisé en Espagne le 14 décembre $1966^{24}$ et des élections législatives de mars 1967 qui donnent de justesse la majorité absolue au Général de Gaulle. Faute de soutien la revue disparaît en mars 1967.

La création de deux écoles françaises à Alicante dans les années 1962 et 1965 , sans subventions officielles et soutenues uniquement par des particuliers expriment la volonté de conserver l'identité française. Murcie, Benidorm et Valence ont vu naître à la même époque d’autres écoles étroitement liées au phénomène pied-noir. L'arrivée à Alicante de centaines d'enfants de formation française provoque de sérieux problèmes dus à la volonté des parents de leur donner un enseignement minimal en français. Des étudiants, souvent les plus âgés, partent en France alors que d'autres, d'origine espagnole, passent dans le système espagnol où ils doivent surmonter le problème linguistique : en effet, bon nombre d'entre eux parlent moins bien le castillan que le français ou le valencien, langue souvent conservée dans les familles levantines d'Algérie.

En 1965 un couple venu de France crée dans un quartier périphérique de la ville la plus petite des deux écoles françaises : celle-ci connaît un certain succès et fait même concurrence au Lycée Français pendant quelques années, mais en raison du manque d'enseignants diplômés elle ne réussit pas à obtenir la reconnaissance du gouvernement français et finit par disparaître au début des années 1980.

La Nouvelle École Française (NEF) fondée en 1962 par des enseignants venus d'Algérie devient rapidement un symbole pour les pieds-noirs ; cependant les autorités françaises se montrent réticentes à « légitimer » un centre dirigé exclusivement par des Français d'Algérie dont bon nombre sont sympathisants de l'OAS $^{25}$. Finalement, en 1972, après des années de négociations la Mission Lä̈que achète l'école ce qui garantit sa permanence et la reconnaissance indirecte de l'État français. Aujourd'hui l'école, devenue le Lycée Français, peut-être considérée comme la réussite de la communauté pied-noir d'Alicante, et ceci malgré les nombreuses difficultés internes et externes de ce centre. Quarante ans après sa création, le lycée est toujours un des établissement les plus réputé de la région ; il a survécu à la relève de la génération d'enseignants piednoirs, et compte actuellement plus de 1200 élèves $^{26}$ et ses installations vont être enfin déplacées sur des terrains plus vastes.

24 Référendum sur la réforme de certaines «lois fondamentales » de l'État franquiste. La proposition de Franco remporta $97.4 \%$ des voix exprimées. Quelques jours avant les piedsnoirs se sont distingué en organisant dans le centre-ville une grande manifestation en faveur de Franco.

25 Il faut signaler que «le discours pied-noir » est totalement absent dans les cours du Lycée Français d'Alicante.

26 Pendant la décennie quatre-vingt dix ce centre a profité de l'arrivée des élèves des lycées français d`Algérie (notamment de ceux d'Oran) qui on pu fuir la crise de leur pays. Il attire aussi une partie des enfants des quelques 2000 fonctionnaires européens de l'Office d'Harmonisation du Marché Intérieur, une institution mineure de l'Union européenne installée à Alicante depuis 1994. Ces circonstances aident à contrecarrer l'effet de l'effondrement démographique que connaît l'Espagne depuis plus d une décennie. Néanmoins. le projet de création d'une école internationale pour les enfants des fonctionnaires européens menace l'avenir du Lycée Français d'Alicante. 
Créée à l'origine pour garantir l'enseignement du français aux enfants piedsnoirs, cette école a réussi à exercer une influence notable ct durable dans la société locale jusqu'au début des années quatre-vingt. En effet, dans le contexte espagnol de ces années, le Lycée Français a été, au delà du simple apprentissage linguistique, le symbole d'une éducation plus libérale, laïcisée et démocratique.

\section{La « deuxième génération » et les Espagnols revenus d'Algérie}

Par leur manière de vivre et leur attachement à la France et à l'Algérie française, les pieds-noirs montrent que souvent ils ne se sont pas facilement intégrés dans la société d'accueil. Cependant ce constat ne peut pas s'appliquer à l'ensemble des exilés établis dans la province. L’âge qu'ils avaient lors de leur arrivée et les contacts gardés avec des Espagnols pendant les années d'émigration en Algéric ont joué un rôle primordial pour leur intégration.

Dès les années cinquante quelques pieds-noirs profitant de la libéralisation économique, du jumelage Alicante - Oran et de la différence du coût de la vie achètent des maisons ou montent des affaires en Espagne. D'autres, sans doute les plus nombreux, avaient encore des proches et des parents dans la région. Même s'ils ont adopté la nationalité française beaucoup d'Oraniéns conservent dans leur milieu une ambiance levantine et restent tout simplement Espagnols à l'égard des autorités espagnoles. En arrivant d'Algérie ils se dispersent dans la capitale et sa province souvent accueillis par leur famille; bon nombre d'entre eux s'installent notamment dans certains villages du nord de la province d'où sont originaires beaucoup d'émigrés espagnols en Afrique du Nord. Ils se sont vite adaptés à la vie en Espagne, leurs relations familiales ont joué dès leur arrivée un rôle important dans leur insertion économique et sociale. En fait, seuls les pieds-noirs n ayant pas de point de chute, ceux qui n'avaient pas de relations dans la région, se sont regroupés dans la capitale et dans d'autres villes du littoral.

En France, une génération après le rapatriement, il est souvent question, dans les colloques et dans les associations, de "deuxième génération pied-noir ". Le traumatisme social, l'importance de la population concernée et l'existence d'un mouvement associatif bien organisé ont favorisé la transmission d'une identité spécifique à la génération qui n'a pas connu l'Algérie. Cette question ne s'est même pas posée pour les enfants de pieds-noirs d'Alicante, même s'ils ont été marqués par l'exil. Ceux qui ont vécu leur adolescence à Alicante et surtout ceux qui sont nés après 1962 sont généralement beaucoup plus ouverts aux influences de la société locale et n'ont pas le même regard sur une Algérie dont ils n'ont pas intériorisé le mythe. Chez. les fils et les filles des pieds-noirs il est difficile de détecter cette ascendance car même s'ils ont grandi dans un milieu particulier ils ne se distinguent pas des autochtones, ils n'ont pas le sentiment d'appartenir à une "deuxième génération pied-noir ». Plus qu'entre la France et l'Algérie française, la question de l'appartenance se pose désormais entre deux réalités beaucoup plus faciles à gérer : la France et l'Espagne. 


\section{CONCLUSION}

La fïn de la Guerre d'Algérie amena sur les côtes levantines plusieurs milliers de réfugiés. Certes beaucoup n’ont fait que transiter dans la région pour se rendre en France, mais un nombre important d'entre eux se sont établis à Alicante et dans sa province. C'est la première fois que la région reçoit un nombre aussi important d'étrangers dans un laps de temps aussi court. Leur nombre n'a jamais été sérieusement déterminé ce qui a contribué à créer le mythe des trente mille pieds-noirs ; en réalité moins de dix mille, dont beaucoup d'inactifs, se sont réellement installés dans la ville. Ces pieds-noirs, souvent défenseurs tenaces de l'Algérie française, ont joué un rôle important dans l'évolution locale, au moment où la ville et le pays s'ouvraient aux influences extérieures après plus de deux décennies d'autarcie politique et économique.

L'existence d'une colonie pied-noire aussi importante en Espagne a éveillé l'intérêt des rapatriés vivant en France pour la province d'Alicante. C'est ainsi que la région devint un marché potentiel pour les entreprises françaises et se transforma en un pôle d'attraction pour les Français en général qui avaient déjà, à plusieurs reprises et à différentes époques, vécu dans la ville. Cette réalité associée aux premiers pas d'un tourisme de masse contribue à la revitalisation de cette ville portuaire en lui rendant son cosmopolitisme traditionnel.

Quarante ans après ces évènements, la plupart des rapatriés de la première génération sont aujourd'hui retraités et leurs enfants sont parfaitement intégrés dans la vie et la société locale. Très visible pendant les premières années, leur empreinte s'est effacée au fur et à mesure de l'évolution de la ville. En effet, le formidable essor de l'économie espagnole des années soixante et soixante-dix a amoindri l'originalité des magasins, des restaurants et des boîtes de nuits pieds-noirs qui avaient monopolisé la scène commerciale. L'évolution de la société qui accompagne l'explosion touristique banalise l'étranger et affaiblit leur image de modernité ; l'arrivée en masse d'autres immigrés nord-européens qui choisissent de prendre leur retraite dans la région attirés par le climat, et, enfin dans une dernière étape, l'arrivée de travailleurs maghrébins sont autant de facteurs qui ont favorisé un changement du panorama migratoire de la région. Aujourd'hui, même s'ils revendiquent leur identité spécifique, même s'ils expriment encore un certain refus de s'adapter à la société locale les pieds noirs ne sont plus considérés comme des étrangers. 


\section{Références bibliographiques}

BONMATÍ ANTÓN (J. F.), La emigración alicantina a Argelia, ed. Universidad de Alicante C.A.P.A, Alicante, 1988.

DUBOIS (C.), "La nation et les Français d'outre-mer : rapatriés ou sinistrés de la décolonisation? " in MIEGE (J. et DUBOIS (C.) Dirs. : L'Europe retrou'ée : les migrations de la décolonisation, l'Harmattan, Paris, 1993. pp. 75-134.

GAVIRIA (M.). El turismo de invierno yel asentamiento de extranjeros en la provincia de Alicante, ed. Instituto de Estudios Alicantinos, Alicante, 1976.

GIMÉNEZ LÓPEZ (E.), Alicante en el siglo XVIII : ecomomía de una ciudad portuaria en el Antiguo Régimen, ed. Institución Alfonso el Magnánimo, Valencia, 1981.

GOZÁLVEZ PÉREZ (V.), « Notas sobre la demografía de la provincia de Alicante », Cuadernos de Geografía, n 11, 1972, pp. 27-77.

HARPO (J.) (MARTÍNEZ GOMIS M.) : "La década prodigiosa " in Batlia (Tres Ciltats Valencianes), $\mathrm{n}^{\circ}$ 7, 1985. pp. 51-62.

HERMET (G.), Les Espagnols en France : immigration et culture, éd. Ouvrières, coll. L'évolution de la vie sociale, Paris, 1967.

JORDI (J.-J.), "De l'accueil au transit : Marseille, centre de redéploiement des rapatriés d'Algérie en 1962 " in KHELLIL (M.) et MAURIN (J.). Les rapatriés d'Algérie én Languedoc Rousillon 1962-1992, éd. Université Paul Valéry-Montpellier III, Montpellier, 1962, pp. 25-35.

JORDI (J.-J.). 1962 : L'Awriéé dés pieds-noirs, éd. Autrement, Paris, . 1995.

JURDAO ARRONES (F.) et SÁNCHEZ ELENA (M.), España, asilo de Europa, ed. Planeta. Barcelona, 1990.

MARTÍNE7. LEAL (J.), "La guerra terminó en Alicante. La tragedia del puerto ". Canclobre $n^{\circ} 7 / 8,1986$. ed. Instituto de Cultura Juan Gil-Albert. Diputación Provincial de Alicante. Alicante, pp. 157-166.

MULA FRANCO (A.). De la immigración a la integración : problemas sociolingüisticos, Thèse de Doctorat dirigée par GIMENO MENÉNDEZ (F.), Departamento de Filología Hispánica. Universidad de Alicante, 1991.

PALACIO (L.). Les pieds moirs dans le monde. éd. John Didier, Rouen. 1968.

PETIOT DE LALUISANT (A.). Rapport à l'Ambassadeur de France à Madrid. La Colonie Franscaise d'Alicante. 21 août 1970, 27 p. (non édité)

QUIÑONERO FERNÁNDEZ (F.), Los inmigrados én la ciudad de Alicante, ed. Universidad de Alicante. Alicante, 1988.

ROMERA ROBERTSON (J.). Estudio descriptivo-explicativo sohre el proceso de imlegracion del colectivo "pied-noir" en Alicante, avril 1992. travail de classe de Sociologic, Université d'Alicante.

SEMPERE SOUVANNAVONG (J.-D.) Los pieds-noirs en Alicante. Las migraciones de la descolonización, ed. Universidad de Alicante, Alicante. 1998.

SEVA LLINARES (A.), Alacant 30 (0)0 "pieds-noirs". ed. 62 S.A., coll. Tres i quatre. Barcelona. . 1968.

SIMON (G.), Géodynamique des migrations internationales dans le monde, P.U.F. Paris, 1995.

STORA (B.). Histoire de l'Algérie coloniale 1830-1954. La Découverte, Paris, 1991.

STORA (B.), Histoire de la Guerre d' Algérie, La Découverte, Paris, 1993.

VALERO ESCANDELL (J. R.), La immigración extranjera en Alicamé, ed. Instituto de Cultura Juan Gil-Albert, Diputación de Alicante, Alicante, 1992.

VILAR (J. B.). Los españoles en Argelia (1830-1914). Centro de Estudios Históricos (CSIC). Murcia 1989. 


\title{
Les pieds-noirs à Alicante
}

\section{Juan David SEMPERE SOUVANNAVONG}

\begin{abstract}
Alicante est un des ports européens les plus proches de l'Afrique du Nord ce qui explique les relations intenses et controversées qu'il a entretenu avec le Maghreb et particulièrement avec l'ouest de l'Algérie. Un des moments les plus complexes de cette relation est la période de l'indépendance de l'Algérie (1962) qui force à l'exil la plupart des Français d'origine européenne qui vivaient dans la colonie. Une petite partie de ces pieds-noirs, la plupart d'origine espagnole, choisissent pour diverses raisons de s'établir en Espagne, souvent dans la province d'Alicante.

La ville d'Alicante est à l'époque une petit capitale de province qui s'ouvre, après plus de deux décennies d'autarcie, au tourisme et aux investissements étrangers. L'arrivée de plusieurs milliers de pieds-noirs consolide l'amorce de la modernisation dans la région. Malgré une intégration pleine de nuances, les pied-noirs ont pendant les premières années qui suivent leur arrivée une influence décisive sur l'économie, la société, et en moindre mesure la démographie de la ville. Ils s'établissent presque dans tous les secteurs de l'économie locale, et se distinguent surtout par le nombre de commerces innovateurs qu'ils ouvrent et par les nouvelles habitudes qu'ils introduisent dans certains secteurs d'une société encore traditionnelle. Après l'arrivée en masse de touristes et de résidents européens (surtout des retraités), à partir des années soixante-dix, l'influence des Pied-noirs diminue. En dépit de cela Alicante conserve toujours d'étroites relations avec la France.
\end{abstract}

\section{The pieds-noirs in Alicante}

\section{Juan David SEMPERE SOUVANNAVONG}

Alicante is one of the closest european harbours to North Africa. This explains why the relationships with the Maghreb, and especially with Western Algeria, have been so intense and controversial. The period of the independence of Algeria (1962) has been one of the most complex in this relationship. It forced most of the french people native of Europe who were living in the colony, to go into exil. Some of these pieds-noirs, most of them with spanish origins, decided to settle in Spain, often in Alicante.

At this time, Alicante is a small province capital which is starting to open to turism and foreign investment, after more than two decades of autarky. The arrival of several thousands of pieds-noirs strengthens the beginnings of modernisation in the region. Despite an integration with a lot of nuances, during the first years, the pieds-noirs have a decisive influence on the town's economy and society, and to a lesser extend, on demography. They establish in almost all the sectors of the local economy and stand out by reason of the innovative businesses thay start as well as by the habits they introduce in some sectors of a society which is still traditional. The massive arrival of turists and european residents (especially retired persons) during the seventies imply a decrease in their influence. However, Alicante still maintain close relations with France. 


\section{Los pieds-noirs en Alicante}

\section{Juan David SEMPERE SOUVANNAVONG}

Alicante es uno de los puertos europeos más próximos del Norte de África lo que explica las intensas y controvertidas relaciones que siempre ha mantenido con el Magreb y en particular con el oeste de Argelia. Uno de los momentos más complejos se da cuando la independencia de Argelia (en 1962) fuerza el exilio de la gran mayoría de los franceses de origen europeo que vivían en la colonia. Una pequeña parte de esos pieds-noirs, de antepasados españoles en su mayoría, eligen por diversas razones establecerse en España, a menudo en la provincia de Alicante.

La ciudad de Alicante es en la época una pequeña capital de provincia que se abre, tras más de dos décadas de autarquía, al turismo y a las inversiones extranjeras. La llegada de varios miles de pieds-noirs dio un fuerte impulso a la incipiente modernización de la región. A pesar de su integración llena de matices los pieds-noirs tienen, durante los primeros años, una influencia decisiva en la economía, en la sociedad y en menor medida en la demografía de la ciudad. Se establecen en casi todos los sectores de la economía local distinguiéndose sobre todo por el gran número de comercios innovadores que abren y por los nuevos hábitos que introducen en ciertos sectores de una sociedad aún tradicional. La llegada en masa de turistas y residentes europeos (sobre todo jubilados) a partir de los años setenta diluye la influencia de los pied-noirs. A pesar de ello Alicante sigue manteniendo estrechas relaciones con Francia. 